\title{
BMJ Open Quality Improving rib fracture management in Morriston hospital: using QI to develop an erector spinae plane catheter service
}

\author{
Tom Rees, ${ }^{1}$ Arthur Ee ${ }^{2}$
}

To cite: Rees T, Ee A. Improving rib fracture management in Morriston hospital: using QI to develop an erector spinae plane catheter service. BMJ Open Quality 2021;10:e000939. doi:10.1136/ bmjoq-2020-000939

Received 7 February 2020 Revised 9 September 2020 Accepted 20 March 2021
Check for updates

\section{(c) Author(s) (or their} employer(s)) 2021. Re-use permitted under CC BY-NC. No commercial re-use. See rights and permissions. Published by BMJ.

${ }^{1}$ Anaesthetics, University Hospital of Wales Healthcare NHS Trust, Cardiff, UK

${ }^{2}$ Anaesthesia, Royal Gwent Hospital, Newport, UK

Correspondence to Dr Tom Rees;

tomorees@gmail.com

\section{ABSTRACT}

Introduction Trauma audit research network (TARN) data for 2018 indicated that we admitted 100 patients with chest wall injuries in our District General Hospital (DGH). Our own retrospective audit of pain team referrals confirmed long length of stay (median 14 days), with $59 \%$ requiring level $2 / 3$ care and $11 \%$ mortality risk. We noticed that Regional anaesthesia was offered to less than $25 \%$ of patients despite $63 \%$ reporting severe pain and decided to introduce an erector spinae plane (ESP) catheter service for rib fractures. Our aims were to reduce length of stay and pain scores.

Methods We set up an email alert system, where TARN data collectors notified us when patients were admitted through the emergency department with rib fractures. Using a secure social media app (Whatsapp), we organised a group of regional anaesthetists who were willing to provide an ESP service. Process mapping and driver diagrams helped to streamline the service.

Results Mean length of hospital stay was reduced from 10 to 7 days after introduction of the service with significant reduction in variability. Mean pain scores improved from 8.9/10 to 5/10 with an average improvement of pain score of 2.78 points on a numerical scale out of 10 . $(n=9)$

Conclusions This service improvement relied on a team of hospital clinicians who agreed to provide an extra pain service for patients with rib fracture. The reduction in LOS may be explained by improved respiratory physiology with ESP catheter placement. The ability to deep breathe, cough and engage in physiotherapy treatment are important factors in recovering from rib fractures. The small reduction in pain scores may be explained by the presence of other injuries. Ongoing improvements in training should improve reliability of catheter placement and reduce practitioner variation.

\section{INTRODUCTION}

Problem description and available knowledge

The trauma audit research network (TARN) data for Morriston hospital documents the admission of just under 100 patients with chest wall injuries in the year 2018. A retrospective audit of pain team referrals confirmed long length of stay (mean 12.4 days), with $59 \%$ requiring level 2 or 3 care, $43 \%$ hospital acquired pneumonia rates and $11 \%$ mortality risk. Audit data also suggested that pain management is poor with under $25 \%$ receiving regional techniques for analgesia, despite $63 \%$ of patients reporting severe pain.

This, together with recent case reports championing the efficacy of a relatively new thoracic wall regional block, drove the development of an rector spinae plane (ESP) catheter service for rib fractures. ${ }^{1}$

\section{Rationale}

Using the Institute for healthcare improvement's model, a select team of regional anaesthetists was set up with an aim to improve the quality of care that patients with rib fracture receive. By reducing pain in these patients, it was hypothesised that length of stay would be reduced.

Quality improvement methodology would be used to improve efficiency in the patient pathway by using driver diagrams and process mapping. Continuous data charts would aim to display changes in patient outcomes.

\section{Specific aims}

The two primary SMART aims were:

- improve analgesia in rib fracture patients by placing ESP catheters by measuring pain scores,

- reduce length of stay in patients with rib fracture by placing ESP catheters.

Data would also be collected to identify any improvements in lower respiratory tract infection (LRTI) rates as a secondary aim.

\section{METHODS}

\section{Context}

Morriston hospital is a 750-bed teaching hospital providing care for a population of 390000 patients. It has one of the busiest emergency departments (ED) in Wales as well as being one of two cardiothoracic surgery centres in the country.

The management of chest wall injuries in Morriston is not streamlined via one specialty or ward. Initial audit data indicated a spread of admitting medical teams with $50 \%$ of patients 
admitted under cardiothoracic surgery, $20 \%$ under orthopaedics and $12 \%$ under general surgery. Referral rates from these specialities to anaesthesia for consideration of regional analgesia were low, with most referrals coming late into a patient's stay via the pain team nurses after all other analgesic options had been exhausted.

Early audit data showed that patients with chest wall injury were not being reliably scored using the blunt chest wall injury pathway published by the trust. Engagement with trauma leads for ED led to an earlier inclusion of regional anaesthesia techniques in the admission flowchart for chest wall injuries coming through ED.

\section{Intervention}

The ESP block is a low risk intervention that has been described as 'paravertebral by proxy'. The exact mechanism of action is not clear, but it is likely that intercostal nerves are blocked with a single mid thoracic injection of local anaesthetic deep to erector spinae muscle. The placement of a catheter into this plane allows infusion of local anaesthetic over a longer period of time. Although the ESP block is simple to perform, its recency in the literature means that few anaesthetists are experienced in placing them in patients. More information of insertion technique and background can be found here: https:// www.youtube.com/watch?v=mG4DRT_4Pjo.

\section{Increasing numbers of trained team members (STEP 1)}

The initial ESP service consisted of pain nurses referring patients with rib fracture to the single advanced regional anaesthetic trainee. The first cycle identified the need for more trained team members to develop the service. More team members were recruited and added to a social media messaging group. This intervention increased the trained anaesthetic team members to 9. Engagement techniques used at this early stage also included raising awareness through presentation at clinical governance meetings. Another recruitment strategy included 'bringa-buddy' training where competent ESP team members could be exponentially increased by training a new anaesthetist each time a team member placed an ESP catheter.

\section{Identifying patients more quickly (STEP 2)}

The process of receiving a regional involved referral through the acute pain team once all other analgesic options had been exhausted. Regional analgesic methods were used only as rescue techniques. Early analgesia using regional techniques was hypothesised to allow earlier discharge through improved ability to deep breathe, cough and receive effective physiotherapy.

The TARN administrators already logged all trauma admissions to the hospital on a daily basis and they agreed to add the email address of the advanced trainee to the alert system used to identify patients with chest wall injury. Process mapping (figure 1) demonstrated the simplification of the referral process with reduction in number of stages from 16 to 12.

Later, pain nurses were added to the messaging group to streamline referrals that were not identified by the TARN alert system.

\section{Making it easier to perform the procedure (STEP 3)}

The performance of ESP catheter insertion relies on a long list of drugs, equipment and documentation. An aide memoir was constructed, with the aim of enabling ESP performance more quickly and efficiently. Identified as an important secondary driver, this included a standardised drug bolus and infusion regime to ensure consistency between practitioners. Subjectively, this made the process of performing ESP catheter insertion

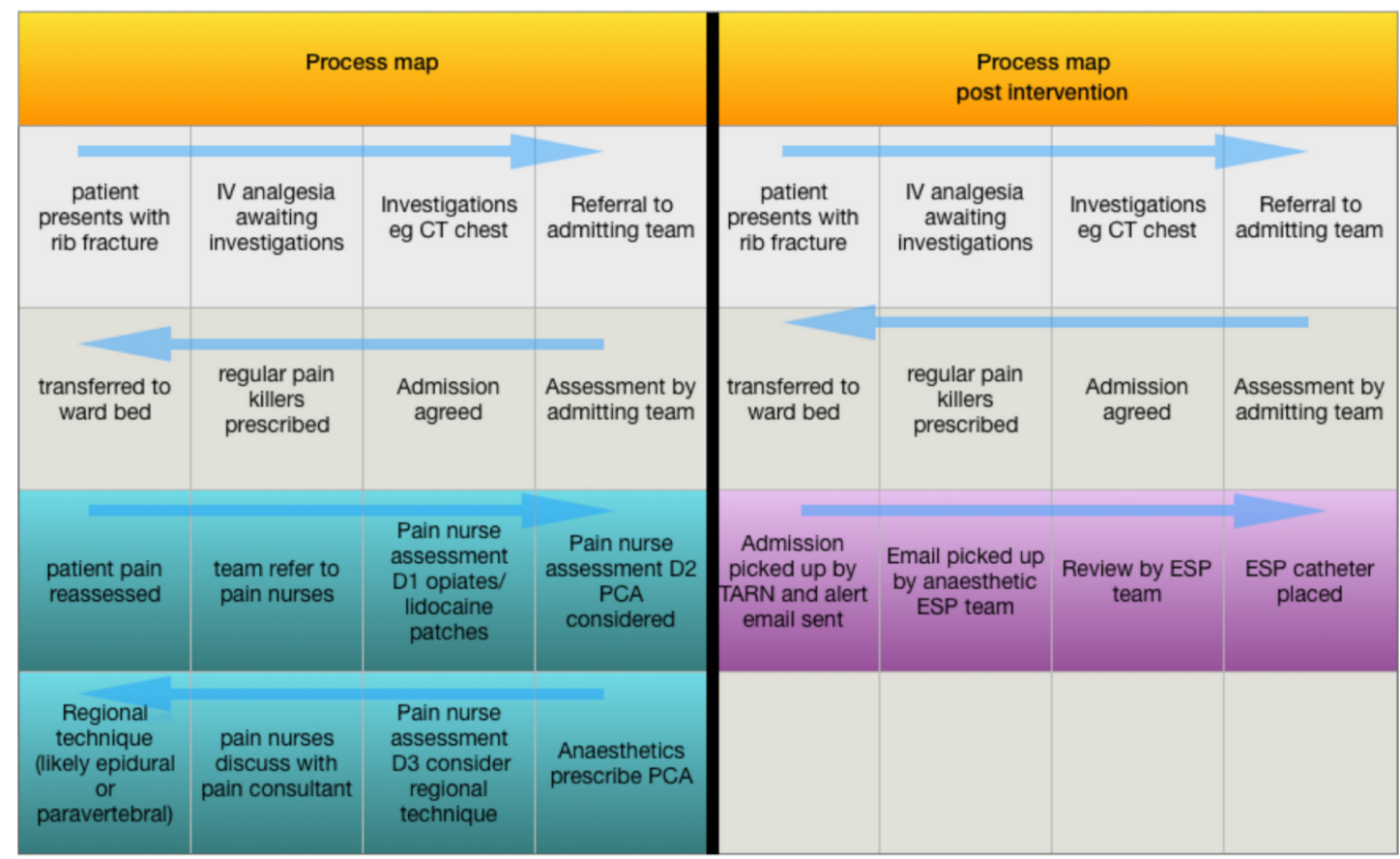

Figure 1 Process mapping preintervention and postintervention. ESP, erector spinae plane; PCA, patient controlled analgesia. 
more efficient, although no distinct process measure was recorded in terms of time taken to collect equipment. Further improvements stemmed from this initial intervention with the organisation of a grab-bag including all paraphernalia required to perform the procedure.

\section{Patient and public involvement}

Patients and public were not involved in the design, conduct, reporting or dissemination of this service setup. Some patients were interviewed to provide feedback in improving the service.

\section{Study of the intervention}

The approach chosen for assessing the impact of the ESP service.

\section{Measures}

The outcome measures chosen were

- Length of stay.

- Pain scores.

- Number of respiratory infections.

The study of the baseline data showed that certain patient cases were obviously outliers in the group. The special cause variation was attributed most commonly to requirement for social care setup before discharge, significant other injuries (as well as rib fractures) and surgical intervention during hospital admission.

This led to 'date of discharge home/transfer to rehabilitation hospital' being adopted as the operational definition if 'ready for discharge' was clearly documented in the case notes. One data point was excluded due to multiple admissions with the same presenting complaint making calculation and comparison of length of stay impossible. The full inclusion criteria in the baseline data are listed below. They were also applied to those patients who received ESP catheters to make the data fair and reliable.

Inclusion criteria for standardised data purposes:

- Unilateral rib fractures.

- No surgical intervention for chest injury.

- No social reason for prolonged stay in hospital.

- Discharged from hospital (not deceased).

- No significant spinal fracture.

- No significant cavity surgery during admission.

- Acute injuries within 2 days of presentation.

- Conscious patients.

Pain scores had been documented in three different ways in the patient's case notes before ESP catheters were introduced. Numerical rating scales out of 3 and 10 were common, as were mild/moderate/severe categorical scales. For the purposes of consistent data display, it was decided to convert pain scores to a numerical scale out of 10. $1 / 3$ became $3 / 10,2 / 3$ became $7 / 10$ and $3 / 3$ became $10 / 10$. Mild, moderate and severe categorical data were similarly converted to ensure the measures had a consistent operational definition across the project and aimed to make the data more reliable.

With LRTI rates occurring with such low frequency, the number of cases between cases of LRTI was used to attempt to display any change post intervention. The use of antibiotics for treatment of chest infection was used as the operational definition of LRTI.

\section{Analysis}

Statistical Process Control (SPC) and run charts were chosen to understand the data and predict future performance of the new system. Continuous data display allowed a more accurate interpretation of whether ESP intervention was causing change in the process. SPCs also allow a visual representation of variation of a system and reduction in variation is a good indicator that care quality has been improved. The continuous charts allowed representation of changes to the service to further pinpoint effective interventions.

\section{Ethical considerations}

All patents were consented for ESP catheter placement. The project was registered with Swansea bay health board clinical audit department.

\section{RESULTS}

The SPC (figure 2) displays preintervention and postintervention data. The data for the first 25 pre-ESP catheter patients shows a mean length of stay of 10.9 days with large variation (32.1-10.2 days).

Intervention using ESP catheters for pain control has reduced the mean LOS to 7.1 days and narrowed the control limits to 12.1-2.1 days. There has been a large reduction in variation in LOS after the ESP intervention. This allows prediction that future LOS using this system would be significantly reduced.

STEP 1 refers to increasing number of trained anaesthetists in the team, an intervention that occurred concurrently with STEP 2 (identifying patients more quickly). STEP 3 refers to construction of an aide memoir and grab-bag, interventions aimed at making procedure performance easier for the team members.

In the SPC for pain scores (figure 3), black vertical arrows represent the same STEP changes as the SPC for LOS. Note that the number of patients included is less than in LOS chart as pain scores were not documented in four patient case notes. One patient who received an ESP catheter had no pain score documented in the baseline data, hence exclusion from the data.

Mean pain scores have been shown to be reduced from 8.9 to $5 / 10$ with the introduction of the ESP catheter service. Average improvement in pain score was $27.8 \%$. The data suggest that an increase in variation as the upper and lower confidence limits has been widened. However, this is because more patients have recorded a lower pain score, indicating an improvement in care.

The data collected were unable to show a change in LRTI rates after ESP intervention. More cases are required to improve reliability of data, yet early trends suggest an increase in the mean number of days between LRTI cases. 


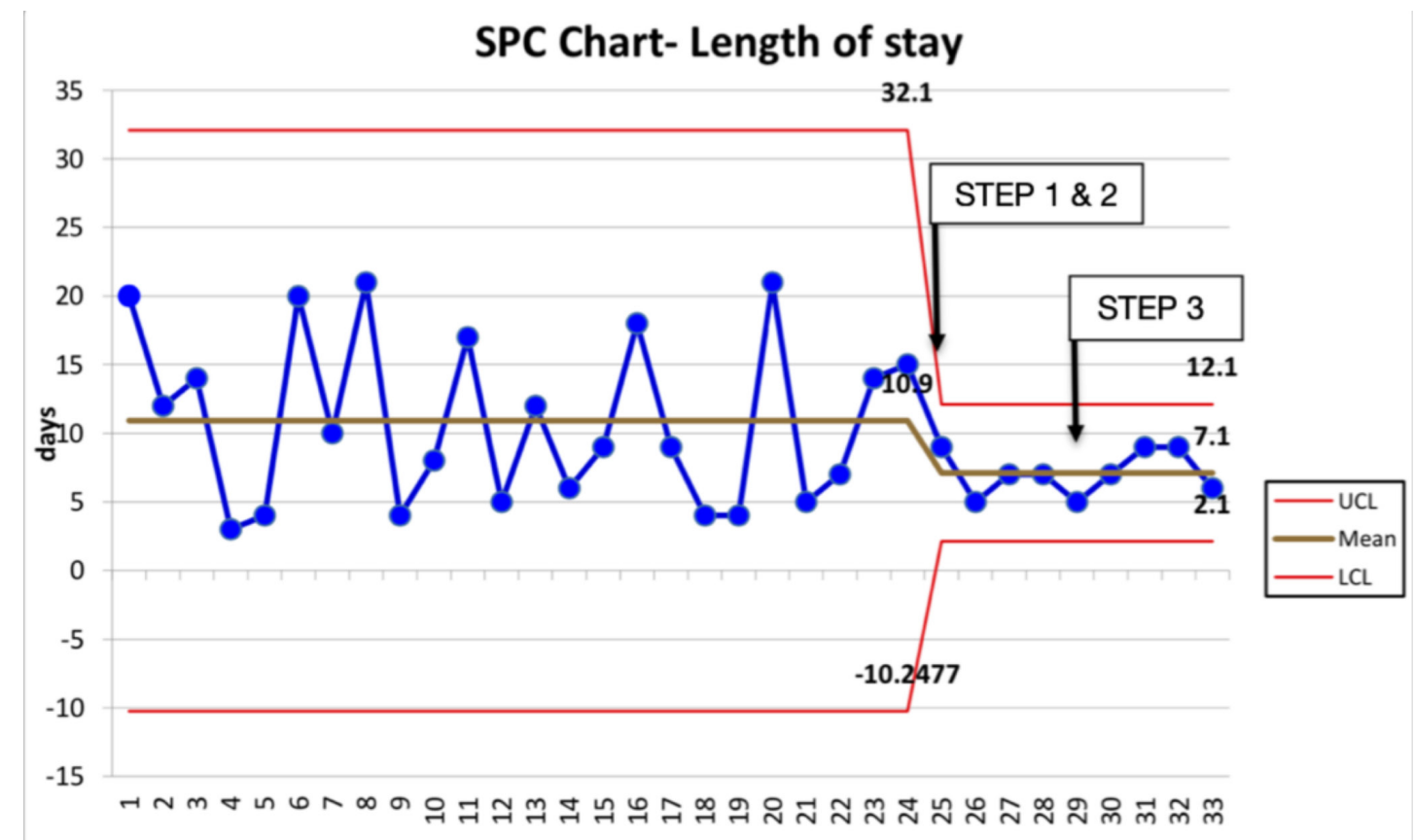

sequential patients

Figure 2 SPC chart of length of stay. UCL, upper control limits.

\section{DISCUSSION}

\section{Summary}

The key findings from the ESP catheter service intervention are that length of stay and reported pain scores are reduced compared with the previous year's baseline data. Variability of the outcome measures were reduced in both LOS and pain scores, meaning that the ESP service improved consistency of quality of care.

\section{Interpretation}

This service improvement project relied on a team of hospital clinicians who agreed to provide an extra pain service for patients with rib fracture in addition to their contracted working responsibilities and roles as anaesthetists in theatres. This service would be made more robust and reliable if sessions for acute pain interventions were organised in job plans for the consultant anaesthetic

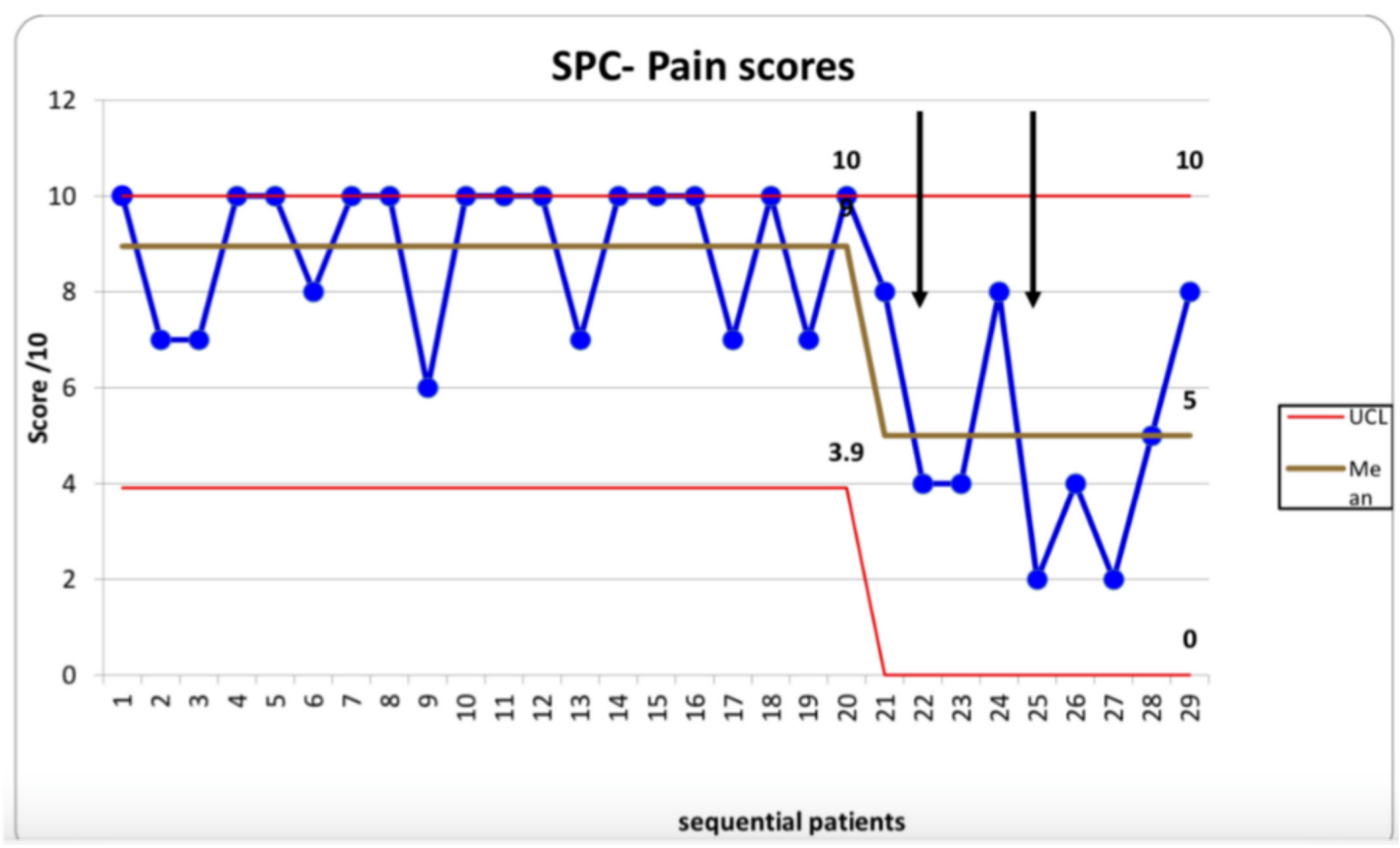

Figure 3 SPC pain scores. 
group. It takes $30-45 \mathrm{~min}$ to site an ESP catheter from consultation and consent, positioning and insertion to dressing and infusion setup.

The mean cost of an inpatient ward bed for blunt trauma in England \& Wales is £281/day. ${ }^{23}$ Analysing this measure alone, this acute pain intervention has saved the Swansea Bay Health board $£ 1067$ per patient admission. The data presented here are not powerful enough to predict reduction in critical care admission, where the mean cost per day is $£ 1328$.

The reduction in LOS may be explained by improved respiratory physiology with ESP catheter placement. The ability to deep breathe, cough and engage in physiotherapy treatment is assumed to improve recovery rates. This theory is supported by recent studies showing improvement in vital capacity after ESP block in rib fracture cases. ${ }^{4}$

The reduction in pain scores is not as drastic as the team had hoped. As stated earlier, this may be due to the presence of other painful injuries not covered by the ESP block. The variation in effectiveness could also be attributed to practitioner performance variability.

Evidence for the reduction in Hospital Acquired Pneumonia (HAP) rates is yet to be presented, and more cases are required for the continuous data charts to suggest any change.

\section{Limitations}

The sample size of this group obviously limits its reliability; however, all ESP catheter patients have had LOS less than the mean LOS for the preintervention group. The continuous data charts suggest this to be a trend that predicts continued improvement in care.

Using LOS as the primary outcome measure was intended to reduce the chance of bias on results that might have occurred when using pain scores alone.

Severity of chest injury was not categorised in this data set, and it may be that the preintervention patients happened to have more serious fractures than the ESP intervention patients. There was no difference in number of ribs fractures before and after ESP intervention, but displaced, fragmented fractures could be more of an analgesic challenge.

A Strength, Weakness, Opportunities, Threats (SWOT) analysis was used to analyse the internal capability of the ESP service. The main identified strength was its quick service setup time. Weaknesses were the time consuming nature of placing an ESP catheter and reliance on an email for early identification of cases. This service was not available out of hours and some concern was raised over the reduced mobility of patients attached to ESP infusion pumps. The opportunities the project allowed were mainly in upskilling the anaesthetic workforce in regional techniques. The threats to the service's continuation include reliance on daily checking of emails by trainees and complex, multidepartmental collaboration.

\section{CONCLUSION}

This service has required intensive effort from the ESP team, and future interventions/Plan, Do, Study, Act (PDSA) cycles should aim to further increase the number of team members who are trained to perform ESP catheterisation. Since these results have been presented locally, the Welsh regional anaesthesia group south have set up a training group to identify next steps in rolling out ESP catheter services for blunt chest wall trauma patients across all sites. Guidance on insertion technique has been distributed to trainees and regional training courses have been organised and run with great success using pig carcass phantoms.

With these early results, it would seem prudent to improve the publicity of this service. This should include further presentations at departmental meetings of ED, anaesthetics and admitting team specialities. This service could potentially spread to other contexts like postoperative pain relief in cardiothoracic surgery and analgesia for intercostal drains on medical wards.

This ongoing project will need to be steered by subsequent anaesthetic trainees to gather more evidence of its effectiveness and capability.

Acknowledgements Many thanks to Dr Simon Ford for guidance in setting up this service and his support in the use of quality improvement methodology. Thanks also to the Regional anaesthetists in Morriston hospital who worked hard to get this service off the ground.

Contributors TR planned the quality improvement project and led the development and maintenance of the service. TR analysed the data and wrote the manuscript. AE conducted the initial audit work and led on data collection throughout the project and helped edit the report.

Funding This project was awarded IQT silver level, a quality improvement training program delivered by Health Education and Improvement Wales (HEIW). The project was a winning entry in the HEIW QIST abstract competition via the LINCymru platform. (HEIW are funding the authorship costs).

\section{Competing interests None declared.}

Patient and public involvement Patients and/or the public were not involved in the design, or conduct, or reporting, or dissemination plans of this research.

Patient consent for publication Not required.

Provenance and peer review Not commissioned; externally peer reviewed.

Data availability statement All data relevant to the study are included in the article or uploaded as supplementary information.

Open access This is an open access article distributed in accordance with the Creative Commons Attribution Non Commercial (CC BY-NC 4.0) license, which permits others to distribute, remix, adapt, build upon this work non-commercially, and license their derivative works on different terms, provided the original work is properly cited, appropriate credit is given, any changes made indicated, and the use is non-commercial. See: http://creativecommons.org/licenses/by-nc/4.0/.

\section{REFERENCES}

1 El-Boghdadly K, Wiles MD. Regional anaesthesia for rib fractures: too many choices, too little evidence. Anaesthesia 2019;74:564-8.

2 Department of Health NHS Reference Costs. 2004 appendix SRC1 NHS trust reference cost index 2004.

3 Christensen MC, Ridley S, Lecky FE, et al. Outcomes and costs of blunt trauma in England and Wales. Crit Care 2008;12:R23.

4 Adhikary SD, Liu WM, Fuller E, et al. The effect of erector spinae plane block on respiratory and analgesic outcomes in multiple rib fractures: a retrospective cohort study. Anaesthesia 2019;74:585-93. 